\title{
Diferente desarrollo cefálico según tipo de conejo
}

\section{Different cephalic development among rabbit types}

\author{
${ }^{1}$ Pere M. Parés-Casanova, ${ }^{2}$ Khelil Sofiane y ${ }^{3}$ Anabel Medina.
}

1 Departament de Ciència Animal, Universitat de Lleida, Av. Rovira Roure 191, 25198 Lleida, Lleida, Cataluña, España. E mail: peremiquelp@ca.udl.cat

2 Laboratory of Reproduction of Farm Animals. Insititute of Veterinary Science. University of Tiaret, Algeria. E mail: khalile.sofiane3@gmail.com

3 Departamento de Ciencia y Producción Agropecuaria. Escuela Agrícola Panamericana El Zamorano, Honduras. E mail: anabel.medina@est.zamorano.edu

Recibido: 15 de mayo de 2018. Corregido: 26 de junio de 2018. Aceptado: 28 de junio de 2018.

Resumen: Como alometría se entiende el crecimiento relativo de una estructura biológica respecto a otra(s) o al organismo total y da cuenta de los cambios de conformación que derivan de la modificación de tamaño. Un caso particular es cuando la velocidad de crecimiento es la misma entre los caracteres en estudio, lo cual recibe el nombre de isometría. A fin de estudiar este fenómeno en el conejo doméstico, se colectó un total de 71 cadáveres frescos de conejos, de diferente edad, correspondientes a animales destetados, y se obtuvo por descarnado el cráneo de cada uno. De ellos, 59 correspondían al tipo de compañía (tipos “toys” y "beliers”) y 12 al tipo carnicero (californiano/ neozelandés). Se captó una imagen digital, en la cara lateral, de cada cráneo, sobre la que posteriormente se situaron 12 hitos anatómicos, analizados mediante métodos de morfometría geométrica. Los conejos de carne presentaron un comportamiento claramente isométrico, mientras que los de compañía, y a pesar de que muchos ya eran adultos, este comportamiento era alométrico. Nos encontramos, pues, ante un caso de gerontomorfia en los conejos de carne - un desarrollo precoz- y una pedomorfia en los de compañía — un retardo en su crecimiento, puesto que, aunque adultos, siguen presentando una cara corta y plana, y un cráneo proporcionalmente grande, caracteres típicamente juveniles-. Para los primeros un desarrollo lo más precoz posible y para los segundos una retención de los caracteres juveniles; en ambos tipos, ejemplo de heterocronías de desarrollo.

Palabras clave: conejo "belier”, conejo “toy”, gerontomorfia, Oryctolagus cuniculus, pedomorfia.

Abstract:The word "allometry" refers to the relative growth of a biological structure to other(s) or to the entire body, and encompasses conformational changes due to size change. When growth is the same among studied traits, it is known as "isometry". In order to study this phenomenon in domestic rabbit, a sample of 71 fresh post-weaned rabbit corpses of different age was collected, and skulls were obtained and defleshed. Of this sample, 59 corpses were from companion type ("toys" and "beliers") and 12 from meat type (Californian/New Zealand) rabbits. From each skull it was obtained a digital picture, on its lateral aspect, and 12 anatomical landmarks were situated on it and analysed by means of geometric morphometric methods. Meat rabbits presented a clear isometric growth, although they were still growing (e. g. have not reached to final growth stage) while companion rabbits presented a clear allometric growth (e. g. adults have not reached a final growth). So, it is illustrated a case of gerontomorphy for meat rabbits -e. g. an early development of skull traits - and a case of pedomorphy for companion rabbits - e. g. a delayed growth in skull traits in order to mantain typical juvenile traits as a short and plain face, and a comparatively big cranium-. In other words, for the former group, a precocious development was detected, while for the latter, it was detected a retention of juvenile traits, being for both types cases of developmental heterochronies.

Keywords: "belier" rabbits, "toy" rabbit, gerontomorphy, Oryctolagus cuniculus, pedomorphy.

Autor para correspondencia: peremiquelp@ca.udl.cat 


\section{Introducción}

En biología, entendemos como alometría el crecimiento relativo de una estructura respecto a otra(s) o al organismo total (Gould 1966) dicho de otro modo, que da cuenta de los cambios de conformación derivados de la modificación de tamaño (Cardini and Polly 2013). El caso particular en que la velocidad de crecimiento entre los caracteres en estudio es la misma se llama isometría (Mitteroecker et al. 2013). Suelen reconocerse 3 clases de alometría: ontogenética, evolutiva y estática (Klingenberg 2016). La primera se refiere a las diferencias de conformación que se van presentando a medida que el embrión crece hasta convertirse en adulto. La segunda comprende las diferencias de conformación morfológica que resultan de los diferentes modos de crecer que tienen los grupos con distintas historias evolutivas. La tercera es el resultado de las anteriores y se evalúa en individuos adultos pertenecientes al mismo grupo etario.

Así, en el conejo, se registra una relación entre el crecimiento corporal y el cefálico, pero cabría preguntarse sobre la relevancia del fenómeno calificado como "pedomorfia" o "pedomorfosis". Esta noción se refiere a la posibilidad de la perduración en vida posnatal de aspectos morfológicos que son limitados al período de desarrollo fetal o de neonatos lactantes. La pedomorfia, en efecto, implica un proceso de retraso del desarrollo ontogenético que acaba conduciendo a una morfología adulta, pero con retención de aspectos llamativos de la morfología infantil (Galán 2010).

A fin de estudiar este fenómeno en el conejo doméstico, se analiza, por métodos de morfometría geométrica, el cambio alométrico del cráneo. Esto se hace en 2 tipos claramente diferenciados: uno, el de compañía seleccionado hacia la retención de caracteres juveniles, o sea, presenta un fenotipo claramente pedomórfico, que lo hace mucho más atractivo a los ojos de los humanos- y otro, como grupo comparativo, el de carne - seleccionado por su precocidad en la producción cárnica-. Con la morfometría geométrica se logra capturar la geometría de la estructura objeto de estudio, lo que significa que esa forma no se describe en términos de sus dimensiones, sino de la relación espacial entre sus partes. La información relativa a la geometría de los objetos se puede recuperar, lo que permite la visualización de los cambios morfológicos (Toro Ibacache, Manríquez Soto \& Suazo Galdames 2010). El método craneométrico utilizado en este estudio se basa en la hipótesis de la matriz funcional, según la cual la morfología del cráneo no depende de un control genético propio, sino de la influencia de las matrices funcionales asociadas. Cada componente funcional se conforma por una unidad esquelética a la que se asocian un conjunto de órganos, tejidos y cavidades, que constituyen la matriz funcional y pueden tener respuestas diferentes a la selección.

\section{Material y métodos}

Se colectó un total de 71 cadáveres frescos de conejos, de diferente edad. De ellos, 59 correspondían al tipo de compañía (animales con caracteres pedomórficos claros) y 12 eran de tipo carnicero (californiano y neozelandés). Los animales se dejaron a la intemperie hasta su total putrefacción y se les cortó posteriormente la cabeza; esta se acabó de descarnar y se maceró con agua, para, finalmente, aclarar el hueso con peróxido de hidrógeno. No se incluyó en el estudio ningún esqueleto con signos de deformación o patologías óseas evidentes. Para el tratamiento de los datos, no se consideró la edad ni el sexo de los especímenes, aunque todos los animales eran ya destetados. La información detallada de los especímenes estudiados, que actualmente se encuentran depositados en el Departament de Ciència Animal de la Universitat de Lleida, puede solicitarse al primer autor. 
Para cada cráneo, se obtuvieron imágenes digitales, en la cara lateral de cada uno, mediante una cámara digital Nikon D1500 equipada con una lente de $40 \mathrm{~mm}$ 1:2.8G MicroNikkor. La cara lateral estaba orientada lo más paralela a la cámara y, a fin de eliminar distorsiones de las posiciones relativas de los ángulos debido a un efecto parallax, la cámara se situó, a la vez, a suficiente distancia para asegurar que el cráneo ocupase únicamente una parte del campo visual, libre de distorsiones. Esta distancia variaba según el tamaño de cada espécimen, pero ello no constituye ninguna traba, al haberse incluido en cada toma un patrón milimétrico, con lo que los tamaños absolutos podían obtenerse por reescalado. Las imágenes eran almacenadas en extensión jpg de unos $9 \mathrm{Mb}$ y luego transferidas al computador. Se situaron 12 hitos anatómicos en cada espécimen (Cuadro 1 y Figura 1). La tipología de los hitos, que se basa tanto en la localización definida de los puntos como en la explicación que pueden dar del fenómeno morfológico en cuestión (Toro Ibacache, Manríquez Soto \& Suazo Galdames 2010), fue de hitos de tipo I (correspondientes a los hitos localizados en la intersección de 3 estructuras, hitos 6, 7 y 9), II (correspondientes a los hitos ubicados en curvaturas máximas, hitos 1, 2, 3, 4, 5, 10 y 12) y III (correspondientes a los hitos situados en extremos cuya definición viene dada por estructuras distantes, hitos 8 y 11). Para consideraciones profundizadas respecto del mapa de hitos y su selección de estos, de acuerdo con los criterios geométricos, revísese Toro Ibacache, Manríquez Soto \& Suazo Galdames (2010). Todo este conjunto de hitos se consideró suficiente para reflejar la morfología en su cara lateral del neurocráneo y del viscerocráneo, y fueron situados en cada imagen mediante el programa TpsDig versión 1.40 (Rohlf 2016). El relevo de los puntos craneométricos fue realizado en 2 réplicas independientes, por la tercera autora. La variación de la forma de los especímenes con el espacio tangente se analizó con el programa TpsSmall versión 1.33 (Rohlf 2015).

Cuadro 1. Descripción de los hitos anatómicos utilizados en este estudio

\begin{tabular}{cl}
\hline Número & \multicolumn{1}{c}{ Hito anatómico } \\
\hline 1 & Punto más rostral del borde alveolar de los incisivos superiores (cara lingual) \\
\hline 2 & Punto más rostral del borde alveolar del primer premolar superior \\
\hline 3 & Punto más caudal del tercer molar superior \\
\hline 4 & Punto más ventral de la bulla timpánica \\
\hline 5 & Punto más caudal de la escama occipital \\
\hline 6 & Proyección del meato acústico externo \\
\hline 7 & Proyección del agujero de la bulla timpánica \\
\hline 9 & Proyección vertical de (9) \\
\hline 10 & Sutura del proceso cigomático del hueso temporal con el arco cigomático \\
\hline 11 & Punto más caudal del borde orbicular \\
\hline 12 & Punto más rostral del borde orbicular en el nivel de (10) \\
\hline
\end{tabular}




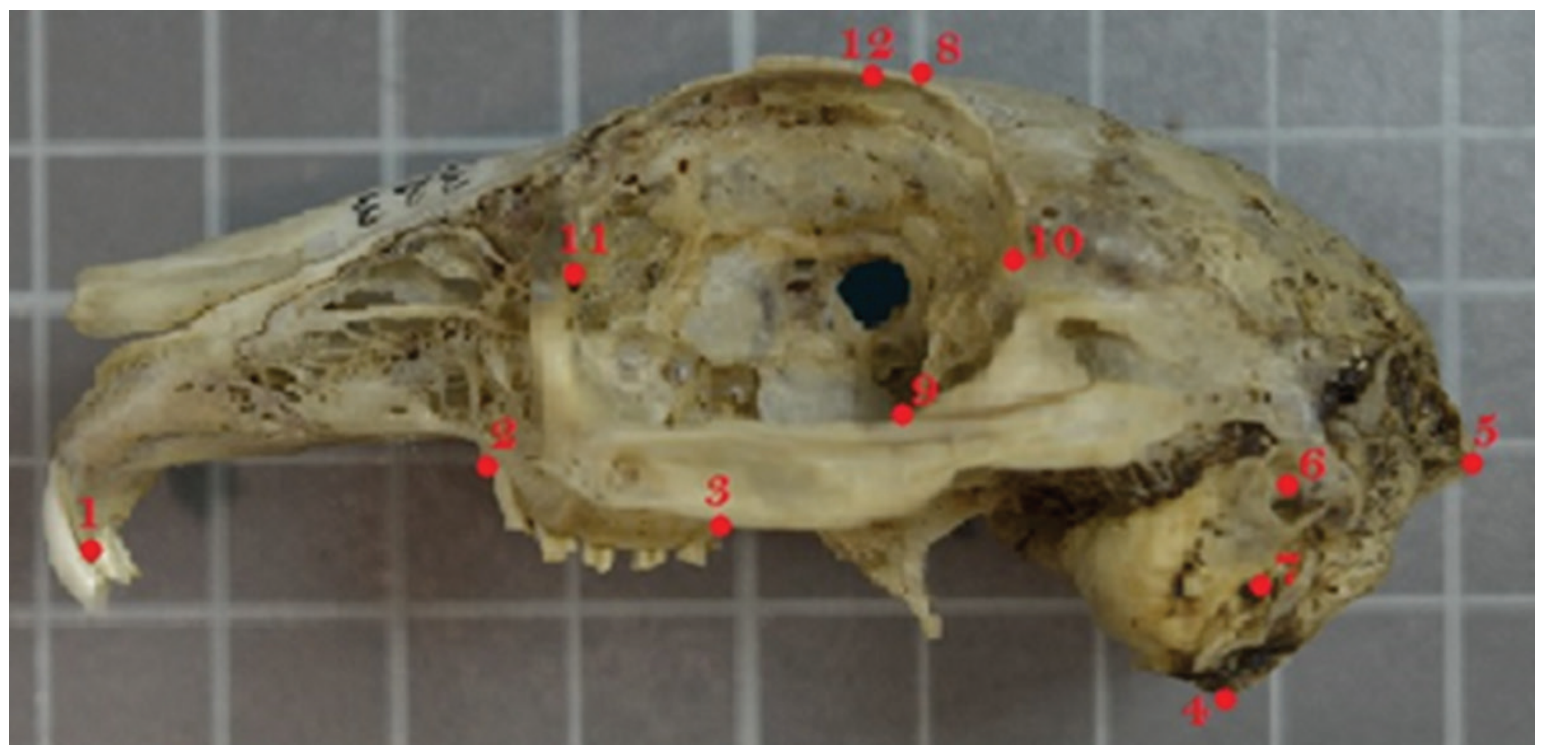

Figura 1. Hitos (12) situados en el plano craneal lateral. Estos se consideraron suficientes para reflejar la morfología en su cara lateral del neurocráneo y del viscerocráneo.

Para evaluar la alometría, se realizó un análisis de covarianzas (ANCOVA) a partir de la regresión lineal simple, utilizando como variable dependiente los valores individuales del primer componente principal (derivado del análisis de componentes principales sobre las variables de conformación, con base en la matriz de varianza-covarianza entre grupos). Una regresión estadísticamente significativa $(p<0,05)$ se consideró alométrica. Los datos se procesaron con el paquete estadístico MorphoJ (Klingenberg 2011). Se estableció para todos los tests un $5 \%$ del nivel de confianza.

\section{Consideraciones éticas}

Los cadáveres procedían de las mismas granjas de cría, donde murieron por causas naturales, no relacionadas con esta investigación, por lo que, al no haberse procedido al sacrificio de ningún animal, no se consideró necesaria ninguna autorización ética.

\section{Resultados}

El análisis de los datos usando el paquete TpsSmall indicó una correlación excelente entre la tangente y el espacio de la forma, lo cual indica la correlación no centrada entre la regresión del espacio tangente, $Y$, y la distancia Procrustes (distancias geodésicas en radianes) con un valor de 1. Ello confirmó que las muestras podían ser analizadas por morfometría geométrica. Dicho de otro modo: aunque la presencia de hitos tipo I era escasa, no se excluía la posibilidad de usar la morfometría geométrica, que permitía la obtención de resultados confiables. El análisis CVA mostró la ausencia de diferencias estadísticamente significativas entre réplicas $(p=0,687)$, por lo que los análisis subsiguientes se realizaron con el promedio de ambas réplicas para cada individuo. Los conejos de carne presentaron un comportamiento 
claramente isométrico (valor predictivo $=10,15 \%, p=0,3254$ ), mientras que los de compañía, una alometría (valor predictivo $=5,84 \%, p=0,0069$ ), siendo los hitos neurocraneales los que mostraban mayor variación. El test ANCOVA corroboró tal diferencia entre estos 2 grupos $(\mathrm{p}<0.0001)$ (Figura 2).

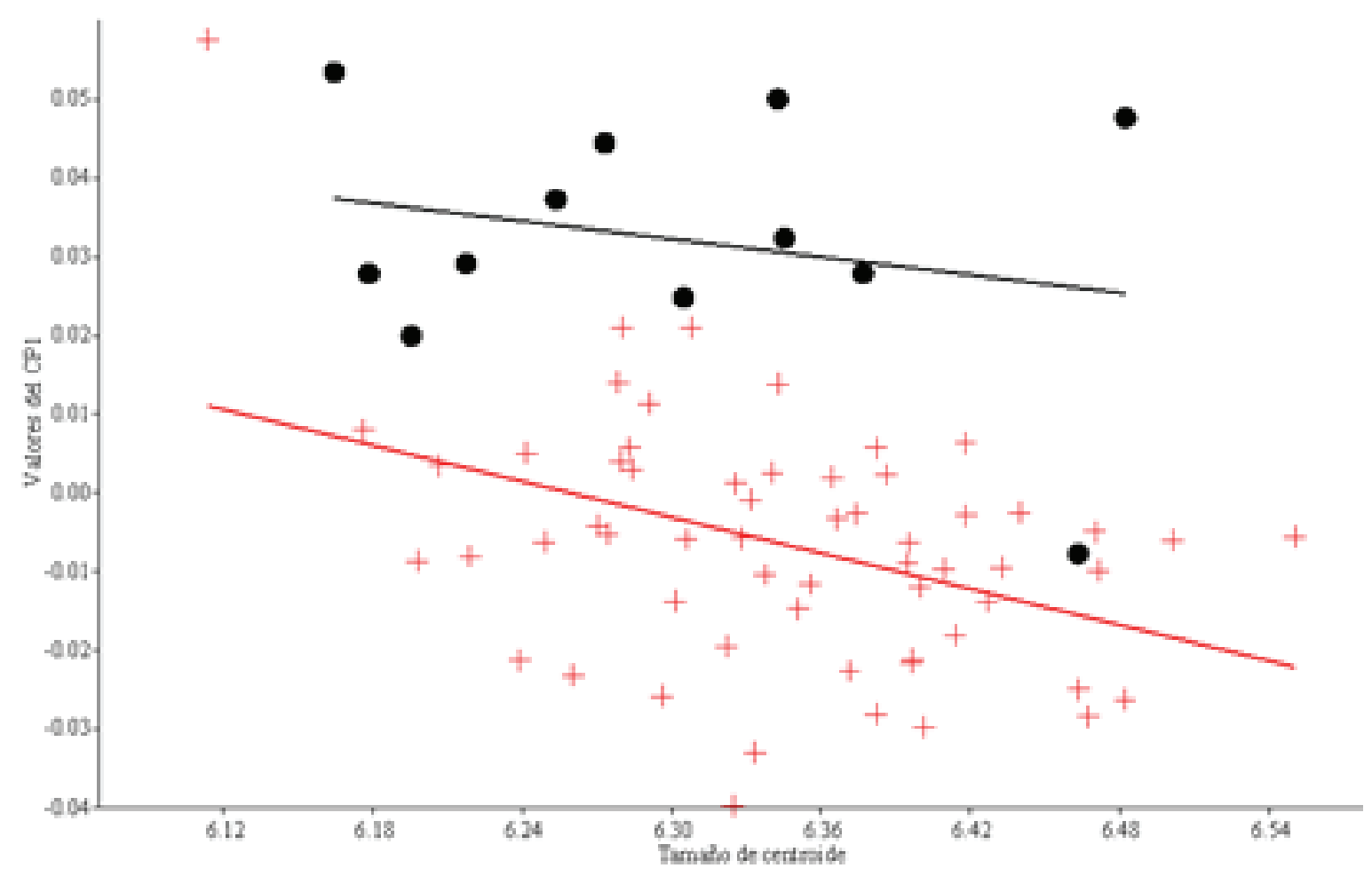

Figura 2. Regresión lineal simple entre conejos de aptitud carnicera (puntos) y de compañía (cruces), utilizando como variable independiente (eje X) el tamaño de centroide (log transformado) y como variable dependiente (eje $\mathrm{Y}$ ) los valores individuales del primer componente principal (CP1). El test ANCOVA corroboró la diferencia entre ambos grupos $(\mathrm{p}<0.0001)$.

\section{Discusión}

El crecimiento corporal o somático es una característica de desarrollo de los organismos que se puede traducir en aumento del tamaño físico y resulta siempre un proceso altamente complejo (Voje et al. 2014; Bolstad et al. 2015). El valor real de crecimiento varía según la especie, puesto que es función de la diferencia existente en el tamaño del esqueleto de aquella, del peso de las crías al nacimiento y de la duración de la gestación (Domínguez-Viveros et al. 2013; Ávila 2017); y puede también variar entre razas o aptitudes (Parés-Casanova, Caballero-Sala \& Perezgrovas 2016), como se demuestra en esta investigación, realizada en diferentes tipos de conejo doméstico. Nos encontramos, así, ante un caso de gerontomorfia en los conejos de carne - un desarrollo rápido (Galán 2010), puesto que se pretende que, en el menor tiempo posible, los animales lleguen al peso deseado- o de pedomorfia en los conejos de compañía - una retención de los caracteres juveniles (Galán 2010), especialmente los faciales-. 
Cabe preguntarse sobre la relevancia de este último fenómeno en los animales domésticos y su posible selección artificial. Esta noción se refiere a la perduración en vida posnatal de aspectos morfológicos limitados al período de desarrollo fetal o de neonatos lactantes. Las nociones de "altricialidad" y "neotenia" se refieren, según algunos autores como Galán (2010), a asuntos separables. La primera se caracteriza por la separación morfológica y conductual entre neonatos, o crías lactantes dependientes, y juveniles autosuficientes, o adultos maduros, que ostentan un conjunto muy diferente de aspectos tanto biológicos como conductuales. La neotenia resaltaría algunas similitudes entre individuos posnatales (fuesen crías lactantes, juveniles o adultos), por un lado, y por otro, fetos o embriones de otras especies cuyos mismos miembros posnatales se parecen menos a sus propios fetos o embriones en determinados aspectos. "Heterocronía" es un concepto que puede englobar las nociones anteriores, así como las de pedomorfia y gerontomorfia. Por lo anterior, aunque en esta investigación nos refiramos únicamente al concepto de "pedomorfia" y "gerontomorfia", lo que se ha detectado son diferentes heterocronías en el conejo doméstico. Y es esta, a conocimiento de los autores, la primera documentación de este fenómeno en el conejo, de lo que se desprende que deberían realizar más estudios en este sentido, encaminado a detectar estas alteraciones del desarrollo en otras razas y especies, y, ante todo, a considerar las consecuencias sobre el bienestar individual que de las heterocronías extremas podría derivarse.

\section{Agradecimientos}

Agradecemos a la empresa CUNIPIC, de Térmens (Cataluña), por su amabilidad en ofrecernos los cadáveres de conejos de compañía, y al matadero MATILDE MASANA, de la Seu d'Urgell (Cataluña), por ofrecernos los neozelandeses y californianos.

\section{Referencias}

Ávila, D. 2017. Variaciones alométricas durante el crecimiento en siete especies de Garzas (Aves: Ardeidae). Rev Biol Trop 65 (4): 1347-1357.

Bolstad, G.H., Cassara, J.A., Márquez, E., Hansen, T.F., van der Linde, K., Houle, D. \& Pélabon, C. 2015. Complex constraints on allometry revealed by artificial Selection on the wing of Drosophila Melanogaster. Proc Natl Acad Sci U.S.A 112 (43): 13284-13289. Doi:10.1073/pnas.1505357112.

Cardini, A. \& Polly, P.D. 2013. Larger mammals have longer faces because of size-related constraints on skull form. Nat Commun 4 (2458): 1-7. Doi:10.1038/ncomms3458.

Domínguez-Viveros, J., Rodríguez-Almeida, F.A., Núñez-Domínguez, R., Ramírez-Valverde, R., Ortega-Gutiérrez, J.A. \& Ruiz-Flores, A. 2013. Ajuste de modelos no lineales y estimación de parámetros de crecimiento en bovinos tropicarne. Agrociencia 47 (1): 25-34.

Galán, C. 2010. Evolución de la fauna cavernícola: mecanismos y procesos que explican el origen de las especies troglobias. Bol Soc Venez Espeleol: 1-31.

Gould, S.J. 1966. Allometry and size in ontogeny and phylogeny. Biol Rev Camb Philos Soc 41 (4): 587-640. Doi:10.1111/j.1469-185X.1966.tb01624.x.

Hammer, Ø., Harper, D.A.T. \& Ryan, P.D. 2001. PAST v. 2.17c. Palaeontol Electron 4 (1): 1-229. 
Klingenberg, C.P. 2011. MorphoJ: An integrated software package for geometric morphometrics. Mol Ecol Resour 11 (2): 353-357. Doi:10.1111/j.1755-0998.2010.02924.x.

Klingenberg, C.P. 2016. Size, shape, and form: concepts of allometry in geometric morphometrics. Dev Genes Evol 226 (3): 113-137. Doi:10.1007/s00427-016-0539-2.

Mitteroecker, P., Gunz, P., Windhager, S. \& Schaefer, K. 2013. A brief review of shape, form, and allometry in geometric morphometrics, with applications to human facial morphology. Hystrix 24 (1): 59-66. Doi: doi.org/10.4404/hystrix-24.1-6369

Parés-Casanova, P.M., Caballero-Sala, M. \& Perezgrovas, R. 2016. Age changes in Chiapas sheep breed according to sex. Rev Investig Vet Peru 27 (4): 651-657. Doi:10.15381/rivep.v27i4.12642.

Rohlf, F.J. 2015. TpsSmall v. 1.33. http://life.bio.sunysb.edu/morph/. http://life.bio.sunysb.edu/morph/.

Rohlf, F.J. 2016. TpsDig v. 2.26. http://life.bio.sunysb.edu/morph/index.html. http://life.bio.sunysb. edu/morph/.

Toro Ibacache, M.V., Manriquez Soto, G. \& Suazo Galdames, I. 2010. Morfometría geométrica y el estudio de las formas biológicas: de la morfología descriptiva a la morfología cuantitativa. Int J Morphol 28 (4): 977-990. Doi:10.4067/S0717-95022010000400001.

Voje, K.L., Hansen, T.F., Egset, C.K., Bolstad, G.H. \& Pélabon, C. 2014. Allometric constraints and the evolution of allometry. Evolution 68 (3): 866-885. Doi:10.1111/evo.12312. 